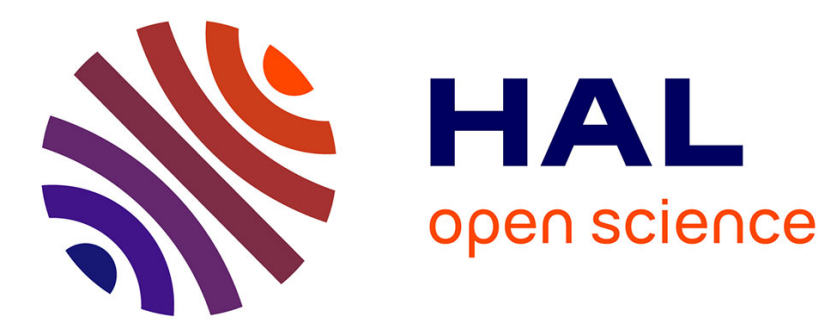

\title{
Le réapprovisionnement continu dans les réseaux industriels, vers une meilleure gestion des interfaces de la supply chain
}

Fouad Jawab, Driss Bouami, Abdennebi Talbi

\section{- To cite this version:}

Fouad Jawab, Driss Bouami, Abdennebi Talbi. Le réapprovisionnement continu dans les réseaux industriels, vers une meilleure gestion des interfaces de la supply chain. La Revue des Sciences de Gestion, 2006. hal-01741705

\section{HAL Id: hal-01741705 \\ https://hal.science/hal-01741705}

Submitted on 23 Mar 2018

HAL is a multi-disciplinary open access archive for the deposit and dissemination of scientific research documents, whether they are published or not. The documents may come from teaching and research institutions in France or abroad, or from public or private research centers.
L'archive ouverte pluridisciplinaire HAL, est destinée au dépôt et à la diffusion de documents scientifiques de niveau recherche, publiés ou non, émanant des établissements d'enseignement et de recherche français ou étrangers, des laboratoires publics ou privés. 


\title{
Le réapprovisionnement continu dans les réseaux industriels, vers une meilleure gestion des interfaces de la supply chain
}

\author{
Fouad JAWAB ${ }^{(1)}$, Driss BOUAMI ${ }^{(2)}$, Abdennebi TALBI ${ }^{(1)}$ \\ ${ }^{1}$ Professeur assistant à l'Ecole Supérieure de Technologie de Fès - Maroc \\ ${ }^{2}$ Faculté des Sciences et Techniques de Fès.
}

\section{RÉSUMÉ.}

La maîtrise des approvisionnements constitue un facteur indéniable pour l'optimisation des flux au sein des réseaux industriels (relation entre pivot et collaborateurs) et entre industriels et distributeurs agissant dans le cadre des relations de partenariat. Dans cet article nous abordons la GPA comme une composante de la chaîne de valeur permettant de dégager un avantage concurrentiel dans le cadre des alliances stratégiques et du réseautage des organisations. Notre champ d'analyse concerne l'interface entre les fournisseurs sous-traitants et les leaders/pivots dans un premier temps (secteur de l'automobile) et entre l'industrie et le commerce ensuite (la grande distribution).

Enfin, nous présentons les résultats d'une étude qui a porté sur les pratiques en matière d'approvisionnement et gestion des interfaces au sein des entreprises marocaines.

Mots-clés : Réseautage, "Supply Shain", ECR, Approvisionnement, Partenariat, Partage.

\begin{abstract}
.
The replenishment constitutes a determining factor in the optimization of Stream among the operators of a same sector, within the framework of the partnership. In this article we approach the Replenishment as a constituent of the chain of value allowing loosening a competitive advantage within the context of the strategic alliances and of the organizations networking. Our Analysis field concerns the interface supplier's andlor industrial subcontractors - Industrial firms (mechanical engineering industries and electronics) and the interface: industrial suppliers - Distributors (the large-scale distribution).

In the end we present the result of the inquest based of the practice in the Replenishment and management the interface for Moroccan's enterprise.
\end{abstract}

KEY WORDS: Networking, supply chain, ECR, Supplies, partnership. 


\section{Introduction:}

Dans un contexte de mondialisation économique et de concurrence de plus en plus agressive, la maîtrise des avantages concurrentiels se révèle d'une importance capitale aussi bien pour les entreprises que pour les réseaux industriels. Dans une approche de gestion en flux tendu, la logistique, longtemps cloîtré dans des opérations de transport et de stockage se voit doter d'une mission stratégique via son potentiel d'amélioration du triptyque qualité, coût et délai (Colin, 1997).

Dans cette optique, les réseaux industriels font appel à des démarches leurs permettant la maîtrise des interfaces de la "supply chain" (SC). Il s'agit, notamment de la démarche ECR (Efficient Consumer Response) et des techniques de réapprovisionnement continu (RC).

L'objectif principal de cet article, est de mettre en évidence le rôle du RC dans l'optimisation des interfaces, avec un regard particulier sur la gestion partagée des approvisionnements (GPA). Nous nous intéressons à son fonctionnement, aux gains générés par sa mise en œuvre ainsi qu'aux positions, enjeux et stratégies adoptées par les différents acteurs.

Dans ce travail, nous abordons respectivement les mouvements qui ont caractérisé le comportement stratégique et structurel des entreprises vers la fin du $20^{\circ}$ siècle, notamment le développement des stratégies relationnelles. Nous évoquons, l'évolution des relations interentreprises vers le partenariat industriel et la restructuration de la concurrence industrielle sous forme de lutte inter-réseaux.

Nous présentons ensuite, la démarche ECR et son rôle dans la synchronisation des flux au sein de la SC, ainsi que l'impacte du système d'information et de communication logistique sur la dynamisation et l'optimisation de ces flux.

Ensuite, nous portons un intérêt particulier aux pratiques et perspectives de développement de la GPA, notamment dans les secteur de l'automobile et de la grande distribution.

Enfin, nous présentons les résultats d'une étude menée auprès de certaines entreprises marocaines afin d'exprimer le niveau de développement des pratiques en matière de la gestion des approvisionnements et de localiser les faiblesses dont souffre la gestion des interfaces amont et aval de ces entreprises.

\section{Adaptation stratégique et structurelle}

\section{Stratégies relationnelles et partenariat industriel}

Face à l'agressivité de la concurrence, les entreprises ont formulées et mis en œuvre des stratégies dites relationnelles ${ }^{(1)}$. Elles sont fondées (Anastassopoulos, 1988) non pas sur la loi de la concurrence, mais sur des relations privilégiées que l'entreprise établit avec certains partenaires de son environnement. Leur objectif, n'est pas de se substituer aux stratégies concurrentielles, mais les deux peuvent soit se combiner, soit se succéder dans le temps. Ensuite, les entreprises ont développé le partenariat industriel qu'on peut définir comme une relation d'affaires (Lambert 1996) bâtie sur mesure et repose sur la confiance mutuelle, l'ouverture, le partage des risques et des bénéfices. L'objectif ultime étant de procurer un avantage concurrentiel résultant d'une meilleure performance d'affaires que ce qu'auraient pu obtenir individuellement les partenaires.

L'opportunité du partage intégrée dans la notion du partenariat a été développé par la théorie des jeux (Baudry., 1994 et Bouvier-Patron., 1994) via la notion du «dilemme du prisonnier» qui considère que dans un jeu à un seul coup, si chaque acteur doit choisir indépendamment de l'autre entre la possibilité de coopérer ou non, il trouvera que la valeur de la tentation de la défection individuelle est supérieure à celle de la récompense de la coopération mutuelle. Il s'ensuit que chaque participant au jeu choisit de ne pas coopérer, croyant qu'il sera seul à agir de la sorte et qu'il gagnera. Il en résulte que le gain global est inférieur à celui qui aurait été

\footnotetext{
${ }^{1}$ Stora. B «Le systéme d’Hermès », Edition HEC 1985. Ces travaux ont été repris par J.P.Anastassopoulos, G.Blanc, J. P.Nioche et B.
} Ramanantsoa dans l'ouvrage « une nouvelle politique d'entreprise » 
obtenu en cas de coopération mutuelle. La seule solution pour éviter ce dilemme est que le jeu se prolonge indéfiniment ou, du moins, que les joueurs pensent qu'il en est ainsi. Dans ce cas, la situation de coopération pour les deux parties est plus avantageuse. Par conséquence, nous estimons qu'au niveau des réseaux industriels, la relation entre les partenaires doit être basée sur un contrat à moyen ou à long terme reconductible et chaque contractant doit avoir un intérêt au maintien de la relation. Les gains générés par cette relation doivent être équitablement répartis et la menace de rupture doit être crédible. A notre sens, la nature de la relation entre deux opérateurs peut être déterminée par deux critères : le degré de confiance et/ou de dépendance, d'un côté et la durée de la relation de l'autre. En conjuguant ces deux facteurs et en s'inspirant des travaux de Roy (2000), nous avons pu obtenir une présentation schématique de l'évolution des relations entre les acteurs vers le partenariat (Figure 1). En fait, si dans une relation d'échange basée sur la sous-traitance de capacité les parties prenantes jouissent d'une grande autonomie, elles ne peuvent avec la sous-traitance de spécialité, qu'entamer une relation de partenariat à cause de la dépendance du donneur d'ordres vis-à-vis du spécialiste. Il a notamment, intérêt à adopter une culture de partage (essentiellement des informations et des ressources) et de créer les conditions favorisant l'émergence de la confiance pour pouvoir bénéficier des compétences de son sous-traitant. Le partenariat devient incontournable avec la sous-traitance d'intelligence, le sous-traitant n'est plus un simple exécutant mais une partie prenante à part entière, échangeant les informations et participant à la recherche et au développement du potentiel technologique du donneur d'ordre afin de satisfaire le client et faire face à la concurrence actuelle et potentielle. En fin, la jointventure permet l'éclosion de nouvelles entités (usines, laboratoire de recherche, réseau de distribution...) concrétisant l'engagement des partenaires.

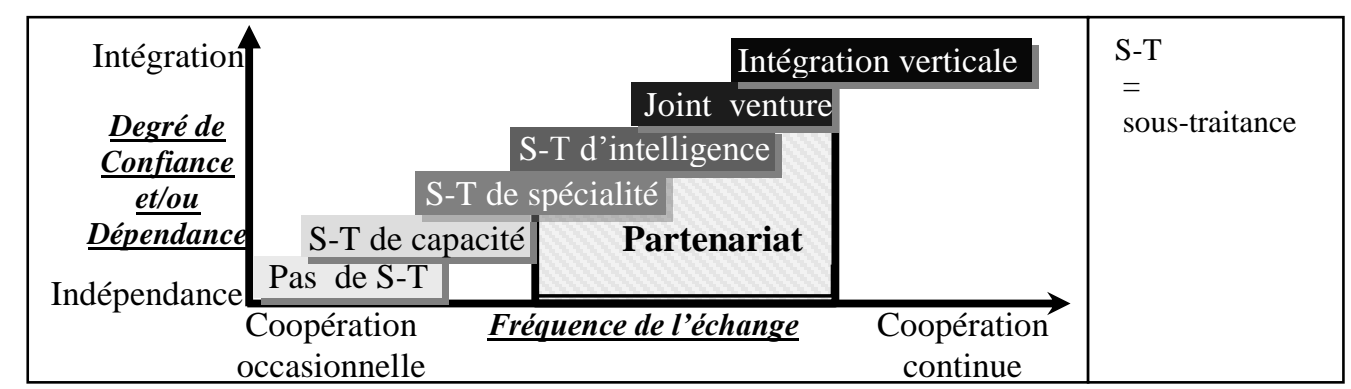

Figure 1: L'évolution des relations d'échange vers le partenariat

\section{Le réseautage conditionne la survie et la performance des organisations. \\ 2.1 Définition et raison d'être des réseaux industriels.}

Juga (1996) défini le réseau comme la configuration concertée d'une variété d'entreprises qui ont établi des relations entre elles et qui cherchent à profiter des synergies potentielles. De son côté, Alcouffe (1999) considère qu'un réseau autorise un nouveau type de relation entre client/donneur d'ordres et fournisseurs/sous-traitant et comprend un leader/pivot qui prend en charge une ou plusieurs activités du réseau, ou assure tout simplement l'articulation des activités des opérateurs spécialisés selon leur compétences.

Le recours à cette forme d'organisation réticulaire peut être justifié par une concurrence dépassant l'entreprise pour se concentrer sur l'ensemble du réseau logistique. En fait, les entreprises ne sont plus directement en compétition entre elles, mais plutôt par l'intermédiaire des divers réseaux logistiques auxquelles elles appartiennent, impliquant ainsi chaque relation client-fournisseur depuis "le fournisseur du fournisseur" jusqu'au "client du client".

La compétitivité des entreprises dépendrait ainsi de leur capacité à s'insérer dans des réseaux performants d'organisations qui mettent en commun leurs compétences et leurs ressources en vue de répondre aux besoins des clients (Hamel et Prahalad, 1995; Giget, 1998). 
En outre, Altersohn (1992) soutient que l'organisation des entreprises en communautés très structurées est nécessaire pour permettre la réunion des conditions propices à une gestion collective des approvisionnements et la concentration sur un espace restreint des différents services dont il faut disposer pour répondre aux attentes de la clientèle.

\subsection{Courants et taxinomie.}

Les soubassements théoriques de cette nouvelle forme d'organisation et/ou de coordination inter-organisationnelle sont loin de faire l'objet de l'unanimité de la communauté scientifique. Nous pouvons en fait, distinguer (Dumoulin., 2000) trois courants des sciences sociales qui se sont focalisés sur l'étude des réseaux :

Pour l'analyse transactionnelle (Richardson, 1972, Williamson, 1985), l'échange s'accompagne d'un coût inhérent à la conception et au suivi du contrat portant sur le transfert du droit d'utilisation d'un bien ou d'un service, au temps passé dans l'échange proprement dit ainsi qu'à l'acquisition de l'information pertinente pour le concrétiser. Cette approche, est basée d'un côté sur l'hypothèse de l'existence d'un degré moyen d'incertitude en raison de la rationalité limitée des agents (Simon H., 1961) et de la nature asymétrique de l'information. De l'autre, sur deux critères déterminant le comportement de la firme, la spécificité des actifs (les caractéristiques de l'investissement) et la fréquence des relations d'échange. En croisant ces deux critères, nous obtenons deux structures de gestion typiques, le marché et la hiérarchie. Au niveau intermédiaire se trouvent des structures qualifiées de formes hybrides, bilatérales et trilatérales, caractérisées respectivement par des relations d'échange fréquentes et occasionnelles. C'est dans cette catégorie, motivée par une baisse sensible des coûts de transaction que l'analyse transactionnelle positionne les organisations en réseau. En s'appuyant sur les mêmes fondements théoriques Thorelli (1986) se démarque en considérant que le réseau possède sa propre logique et il n'est pas le résultat du compromis entre marché et hiérarchie ou une forme altérée de l'un ou de l'autre. Selon l'auteur, un réseau est constitué de deux firmes (ou plus) liées par des relations d'échange suffisamment fortes pour créer une sorte de sous-marché contractuel dans le marché global. Ces relations d'échange s'inscrivent dans la durée et leur stabilité exige un certain nombre d'accord minimum, ainsi qu'une confiance réciproque entre agents. Un effort de coordination est crucial, faute de quoi il risque de ne plus y avoir adéquation entre les différents plans d'action.

Quant au courant sociologique, l'avènement des structures réticulaires n'est pas le résultat du seul calcul d'optimisation économique, mais aussi, et surtout le fruit des relations entre les organisations et entre les individus qui les composent (Gulati, 1998). Les interactions entre les organisations d'un côté et entre les individus de l'autre développent des transactions humainement satisfaisantes plutôt qu'économiquement optimales. Pour cette approche, l'émergence du réseau est une réaction aux insuffisances de la bureaucratie. En fait, l'inadaptation des mécanismes du marché pour la régulation des échanges a permis à la culture, aux normes et aux valeurs (le clan) de devenir une forme de gouvernement de l'entreprise à part entière, à l'instar du marché et de la hiérarchie.

En fin, le courant stratégique (Miles et Snow., 1984 et 1992), considère que l'émergence du réseau n'est rien d'autre que la résultante de la modification de l'environnement des entreprises engendrée par la quête de position concurrentielle dominante via la mise en place des stratégies de recentrage. En fait, à partir des années 80 , on a assisté à un mouvement de recentrage (Paché, 1993) des organisations qui consiste d'abord à se focaliser sur quelques maillons de la chaîne de valeurs (recentrage sur le métier) et ensuite à se focaliser sur les activités jugées indispensables pour la satisfaction des besoins et attentes de la clientèle (recentrage sur la mission). Si ce mouvement de "retour à l'essentiel" s'est traduit par une prise en charge externe des activités de gestion des flux physiques et informationnels par les 
prestataires logistiques, il a permit à la fois une désintégration des activités logistiques et une "réintégration" sous une forme réticulaire.

La conjugaison de ces différents courants par Dumoulin (2000), lui a autorisé la formulation d'une définition synthétique : «le réseau n'est ni quasi-firme, ni quasi-marché, mais une forme transactionnelle à part entière, il est un ensemble contractuel organisé, articulé associant au minimum deux partenaires liés par des relations d'échanges à plus ou moins long terme et par le sentiment d'appartenance à une entité collective. Il résulte d'une action spécifique d'organisation et de régulation fondée sur des modes plus ou moins formalisés et élaborés de contrôle, ainsi que sur la confiance ».

Par ailleurs, s'il est pratiquement impossible de cerner l'ensemble des formes réticulaires, nous avons pris l'initiative de distinguer et de schématiser trois formes type de réseaux (Figure2). La "firme - réseau", appelée également "firme en réseau" (Paché, 1993) ou encore l'entreprise étendue (Benchimol, 1993 et Alban 1997) régies d'une façon contractuelle et caractérisée par la mise en commun de ressources complémentaires entre un leader et des sous-traitants. Le «réseau de firmes», coordonné par une firme/pivot et composé de concurrents ayant une logique de partage ou de mise en commun de ressources identiques, et dont l'effet de taille constitue le principal avantage distinctif (pouvoir de négociation avec les partenaires, économies d'échelle et capacité d'investissement).

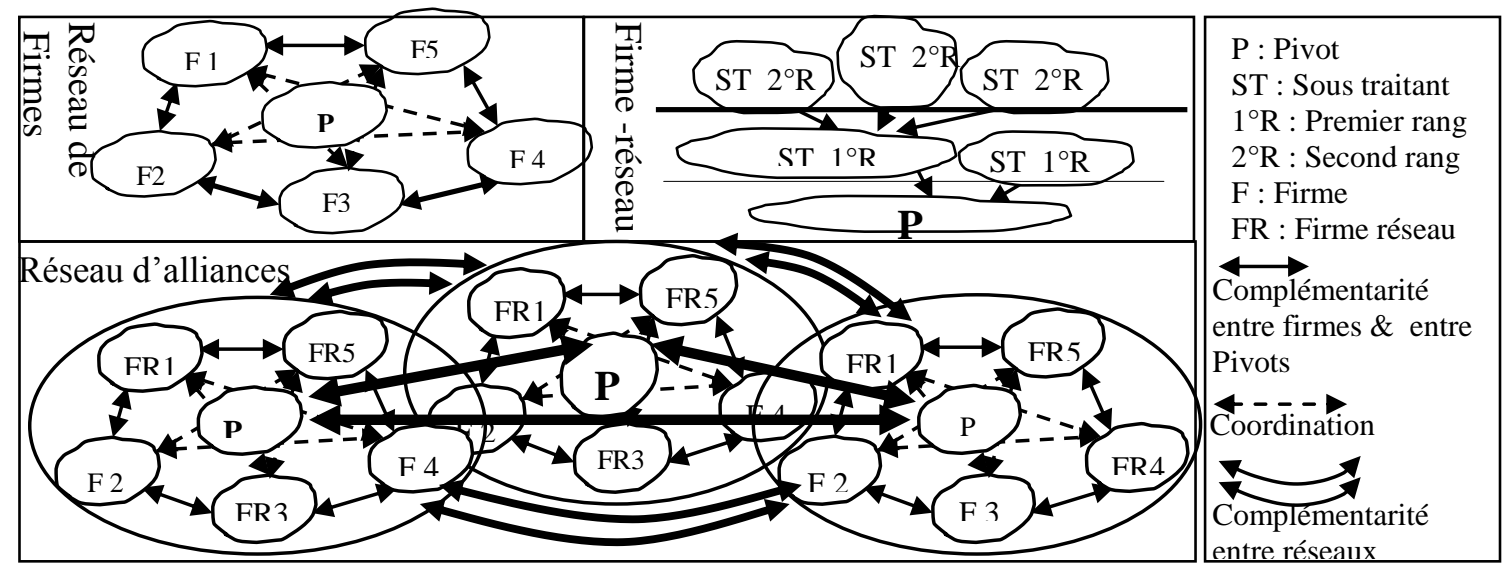

Figure 2: Les trois types de réseaux

Enfin, le réseau d'alliances qui résulte de la combinaison des logiques de partage et de complémentarité des ressources. Régi par une firme - pivot, ses avantages sont plus le résultat d'une incrémentation et d'un enrichissement mutuel que d'une simple addition, (synergie du réseau). Son atout majeur c'est l'«effet boule-de-neige», particulièrement utile dans le contexte des industries émergentes où plusieurs technologies s'affrontent (Tableau 1).

\begin{tabular}{|l|c|c|c|}
\cline { 2 - 4 } \multicolumn{1}{c|}{} & Firme -réseau & Réseau de firmes & Réseau d'alliances \\
\hline Mode de coordination & contrat & contrat & contrat \\
\hline Ressources & complémentaires & identiques & Complémentaires et identiques \\
\hline Rôle de leader & Donneur d'ordre & coordonnateur & coordonnateur \\
\hline Effets / avantages & $* * * *$ & Taille & boule-de-neige \\
\hline composants & Sous traitants & concurrents & firmes en réseau \\
\hline
\end{tabular}

\section{L'ECR et le système d'information logistique, quelle dynamique pour les réseaux? 1. Montée en puissance de la démarche ECR}

Née aux Etafs-Unis en 1987 . Ia Réponse. Efficace au Consommateur "Efficient Consumer Response" (ECR) cherche à exploiter les gisements de rentabilité qui résident dans les relations industriels-distributeurs (coûts d'interface). En fait, elle consiste à assurer une compatibilité organisationnelle et informationnelle totale entre les systèmes logistiques des 
fabricants et ceux de leurs clients distributeurs. De manière idéale, les sorties de caisse vont déclencher d'aval en amont une série d'opérations en vue d'assurer le réassortiment des linéaires dans les meilleures conditions de coût et de service. L'objectif ultime étant de satisfaire le consommateur final en lui offrant le bon produit, au bon moment et au bon prix. La démarche ECR est articulé, essentiellement, autour de quatre éléments, la gestion de la demande (optimisation de l'activité promotionnelle et introduction des nouveaux produits), la gestion des approvisionnements, les technologies de support et les intégrateurs tel que le CPFR "Collaborative Planning, Forecasting and Replenishment" traduit ( Paché 2000) par Système Coopératif de Planification, Prévision et Réapprovisionnement (SCPPR) et dont nous avons schématisé les composantes et l'impact (Figure 3).

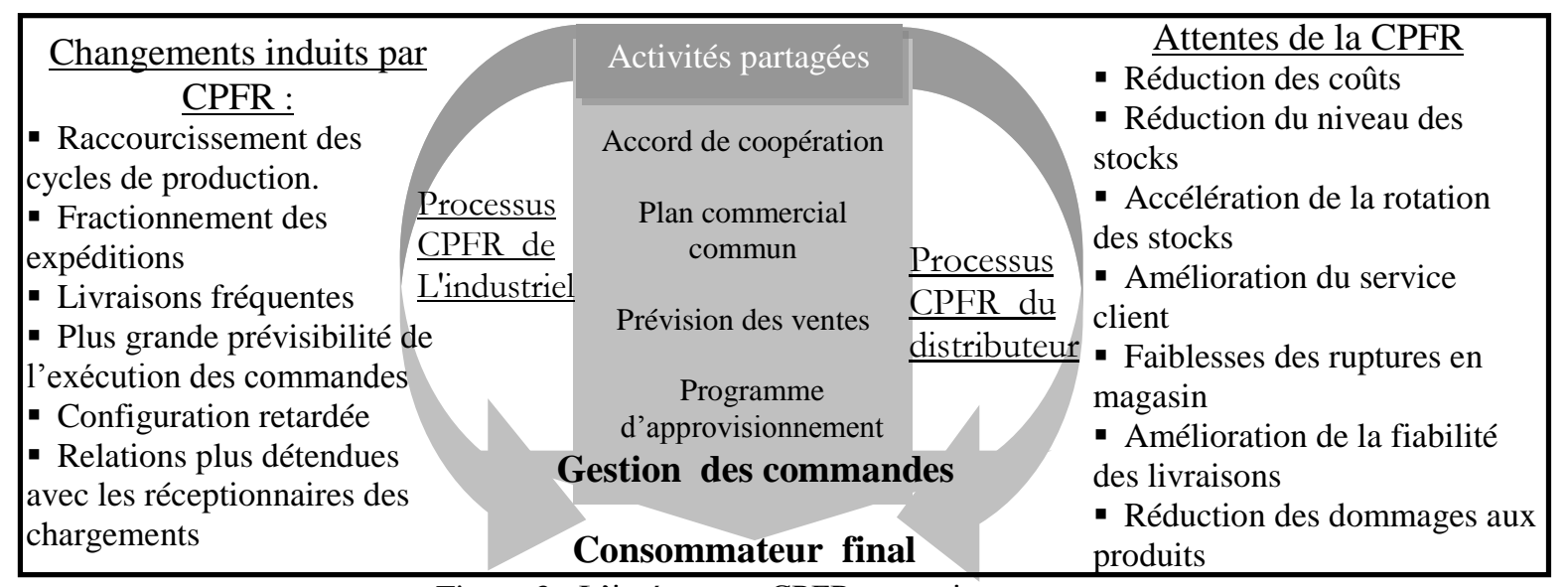

Figure 3 : L'intégrateur CPFR et son impact

\section{Mise en ouvre et perspectives de l'ECR}

La démarche ECR ne cesse de prendre de l'ampleur dans les relations industrie-commerce grâce notamment, aux gains qu'elle autorise. A titre d'exemple, la mise en œuvre en France des concepts ECR en 2000 (Gallo, 2002), a permis à 100\% des adhérents de passer leurs commandes en EDI, à 57\% d'établir leur factures par EDI et à $70 \%$ de passer en mode GPA.

L'ECR a aussi une dimension stratégique permettant aux collaborateurs une coordination pouvant aller du diagnostic stratégique jusqu'à la mise en place des objectifs communs. A cet égard, la coopération entre Système U et Danone portant sur la catégorie "eaux" (Tableau 2) est illustrative.

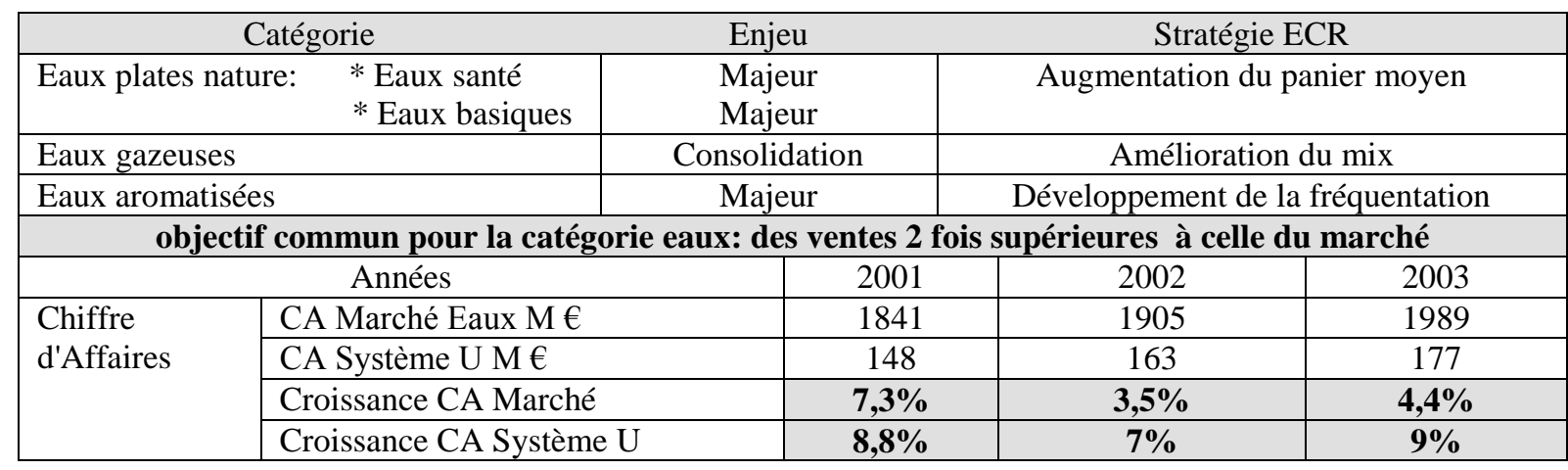

Source: Jouanneau $2002 \quad$ Tableau 2 : Synthèse du diagnostic stratégique partagé et objectif commun

Toutefois, le développement de cette démarche est entravé ${ }^{(2)}$ par l'insuffisance d'engagement des directions générales, le manque de confiance sur le partage d'informations chiffrées, les

\footnotetext{
${ }^{2}$ Difficultés exprimées par les participants à la conférence officielle ECR France 2002
} 
attitudes et les comportements trop axés sur le rapport de force et le manque de visibilité des résultats ECR à cause de manque d'indicateurs partagés et d'objectifs communs chiffrées. Pour de meilleures perspectives, un engagement des directions générales sous forme de charte ECR et la mise en place des indicateurs chiffrés, partagés et simples (Chiffre d'affaires, taux de service, nouveaux produits, promotions...) sont indispensable.

\section{3. le développement des systèmes d'information et de communication logistiques}

Selon N. Fabbe-Costes (1998) le système d'information est doté d'une place de choix dans le management de la chaîne logistique. Trois raisons au moins (Lievre, 2000) peuvent expliquer une telle situation. Tout d'abord, la synchronisation des flux physiques et informationnels est reconnue comme le principe de base du processus logistique. En suite, c'est le flux d'information qui permet généralement de piloter le flux physique. La logistique n'est - elle pas devenue, à partir des années 90, une technologie de maitrise des flux d'information et de marchandise? (Colin, 1996); en conséquence, les flux informationnels prennent le devant par rapport aux flux physiques. Enfin, la maîtrise du système d'information devient un enjeu majeur pour la démarche logistique. Ainsi, les progrès des Systèmes d'Information et de Communication Logistiques (SICL) vont largement contribuer à l'évolution de la démarche logistique elle-même. Dans un objectif de synthétisation, nous avons élaboré un schéma (Figure 4) représentant, à notre sens, les principaux objectifs, les outils, les composantes, les contraintes et les acteurs du SICL. Quant au rôle de ce système au niveau de la gestion des interfaces, notamment la maîtrise des approvisionnements (Figure 5), des solutions ont été développés ses dernières années et dont on peut citer le "GPA MANAGER" qui se prête dans le cadre des stratégies de collaboration industrie-commerce.

\begin{tabular}{|c|c|c|c|c|c|c|}
\hline \multicolumn{3}{|c|}{ Composantes intrinsèques à la logistique } & Référentiels & \multicolumn{2}{|c|}{ Composantes analytiques } & CALS: Computer aided \\
\hline \multicolumn{3}{|c|}{$\begin{array}{c}\text { Niveau d'activité, utilisation des ressources, } \\
\text { Arbitrage : coût niveau de service }\end{array}$} & Temps, secteur & \multicolumn{2}{|c|}{ Réalisations, objectifs, écarts } & $\begin{array}{l}\text { Support or Continuous } \\
\text { Acquisition at Lifecycle }\end{array}$ \\
\hline \multicolumn{6}{|l|}{$\longrightarrow$} & IWSDB : Integrated \\
\hline \multirow[t]{2}{*}{$\begin{array}{l}\text { Gestion des } \\
\text { flux des/avec } \\
\text { les partenaires : } \\
\text { clients, } \\
\text { fournisseurs et } \\
\text { prestataires }\end{array}$} & \multirow{5}{*}{$\begin{array}{l}\text { Progiciel de } \\
\text { modélisation } \\
\text { de réseaux } \\
\text { logistiques; } \\
\text { EDI ; } \\
\text { CALS ; } \\
\text { Ingénierie } \\
\text { concourante ; } \\
\text { Progiciels } \\
\text { de gestion } \\
\text { intégrée : } \\
\text { ERP, APS, } \\
\text { SCM, EAI, } \\
\text { MES }\end{array}$} & \multirow{5}{*}{\multicolumn{2}{|c|}{$\begin{array}{l}\text { EDIFACT/EANCOM } \\
\text { GALIA/ODETTE } \\
\text { ALLEGRO (code à barre). } \\
\text { IWSDB, } \\
\text { Norme ISO 8879SGML, } \\
\text { Norme ISO 8632 CGM, } \\
\text { Norme ISO } \\
\text { 8613/7CCITT... } \\
\text { Logiciel de gestion du } \\
\text { transport RAO; } \\
\text { Logiciel de gestion } \\
\text { des stocks et des } \\
\text { achats ; } \\
\text { Logiciel de gestion des } \\
\text { nomenclatures ; } \\
\text { Logiciel de prévision } \\
\text { de la demande; } \\
\text { Logiciel de gestion } \\
\text { d'entrepôt ; } \\
\text { Logiciel de gestion de } \\
\text { la production..etc }\end{array}$}} & $\begin{array}{l}\text { Ouverture sur } \\
\text { les systèmes } \\
\text { d'information } \\
\text { des partenaires }\end{array}$ & \multirow{5}{*}{$\begin{array}{l}\text { Direction } \\
\text { générale ; } \\
\text { Direction } \\
\text { logistique ; } \\
\text { Directions } \\
\text { fonctionnelles ; } \\
\text { Système } \\
\text { opérationnel ; } \\
\text { Partenaires : } \\
\text { (clients, } \\
\text { fournisseurs); } \\
\text { Prestataires : } \\
\text { (transporteur, } \\
\text { entreposeur.) }\end{array}$} & \multirow{6}{*}{$\begin{array}{l}\text { Metafile } \\
\text { 8613/7 du CCITT : norme } \\
\text { pour les échanges } \\
\text { d'informations graphiques } \\
\text { en mode trame; } \\
\text { APS :Advanced Planning } \\
\text { \&Scheduling systems } \\
\text { SCM: Supply Chain } \\
\text { Management } \\
\text { EAI : Entreprise } \\
\text { Application Integration. } \\
\text { MES : Manufacturing } \\
\text { Execution System }\end{array}$} \\
\hline & & & & \multirow{2}{*}{$\begin{array}{l}\text { Intégration } \\
\text { au système } \\
\text { d'information } \\
\text { global de } \\
\text { l'entreprise }\end{array}$} & & \\
\hline \multirow{2}{*}{$\begin{array}{l}\text { Anticipation } \\
\text { des flux à } \\
\text { l'intérieur } \\
\text { de } \\
\text { l'entreprise : } \\
\text { prévision, } \\
\text { expression des } \\
\text { besoins... }\end{array}$} & & & & & & \\
\hline & & & & \multirow{2}{*}{$\begin{array}{l}\text { Interfaçage } \\
\text { avec des } \\
\text { applications } \\
\text { logistiques } \\
\text { spécifiques }\end{array}$} & & \\
\hline $\begin{array}{l}\text { Suivi des } \\
\text { flux à } \\
\text { l'extérieur } \\
\text { de l'entreprise }\end{array}$ & & & & & & \\
\hline Objectif & Outils & \multicolumn{2}{|c|}{ Composants } & Contraintes & Acteurs & \\
\hline
\end{tabular}

Figure 4 : Quelques composants du système d'information et de communication logistique (SIGL)

En s'appuyant sur un traducteur EDI multinorme (EDIFACT, GENCOD, XML...) et multi réseau (ATLAS400, ALLEGRO...), le progiciel permet au fournisseur de maitriser les stocks 
de ses clients et de générer lui même ses propositions de commande qui seront envoyées ensuite aux clients afin d'effectuer le réapprovisionnement. Parmi ses fonctionnalités, nous distinguons l'anticipation des flux produits, le lissage des promotions, la réduction des stocks et des ruptures en entrepôts et magasins et l'optimisation des chargements des camions.

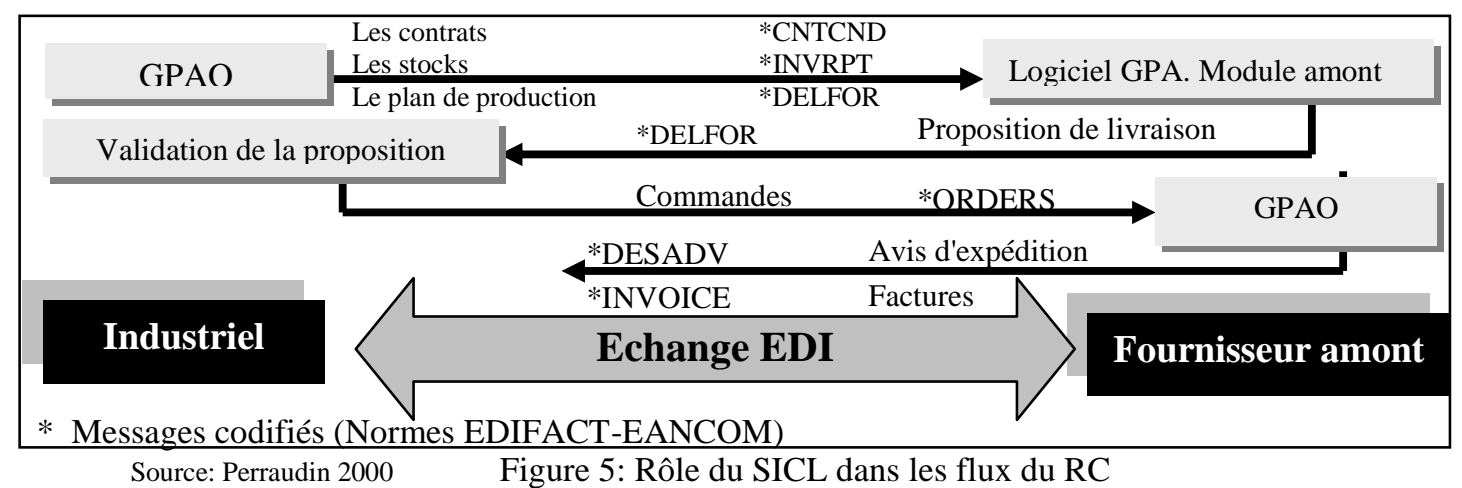

\section{III.Le réapprovisionnement continu au service des réseaux industriels}

\section{Les techniques du RC}

En matière de RC, l'approche de point de commande a été remplacée par une autre désignée par l'acronyme CRP "Continuous Replenishment Program" (Martin, 1998). Cette nouvelle approche a été testée d'abord cher Heinz Canada où les besoins des clients peuvent être visualisés sur un horizon de 26 semaines. Dans le cadre de cette approche, deux stratégies se manifestent (Figure 6). D'abord, un RC géré par le détaillant connu par Retail-Manager Replenishment $(R M R)$. Il permet d'intégrer des données supplémentaires relatives à l'environnement du point de vente et du réseau de distribution, telles que les opérations commerciales et les capacités internes du détaillant (réception, entreposage, expédition). L'incertitude du processus de réapprovisionnement est ainsi diminuée puisque le maillon où est concentré le pouvoir de décision côtoie le consommateur final. Ici, le détaillant est responsable de l'approvisionnement de ses propres sites. Il élabore les prévisions, détermine ses propres besoins de réapprovisionnement, crée les ordres d'achat, et les transmet à ses fournisseurs. Ensuite, un RC géré par le fournisseur et connu généralement par GPA "Vendor-Managed Inventory" ou "Replenishment" (VMI ou VMR).

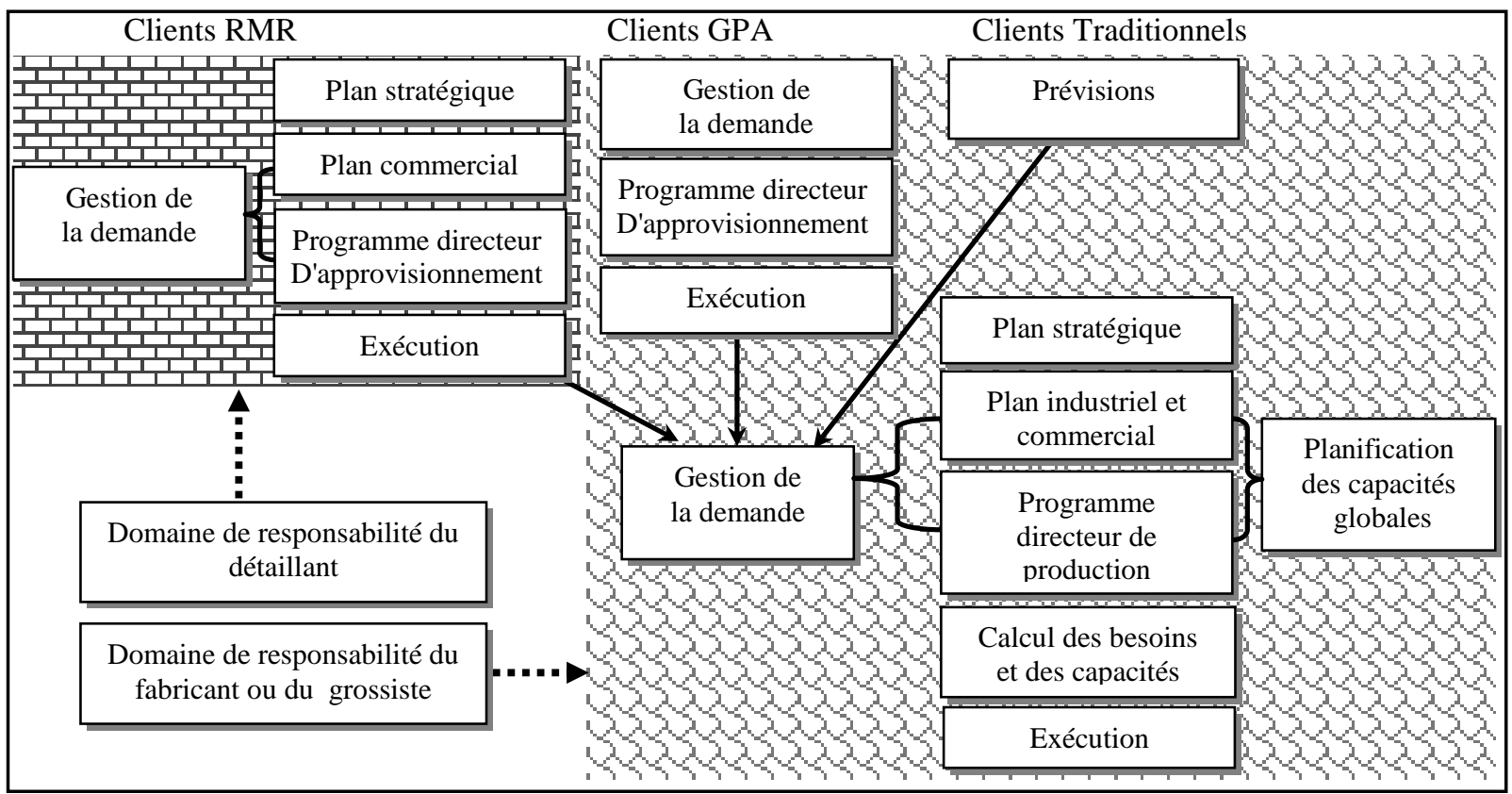




\section{Le rôle des fournisseurs dans la SC.}

La GPA, fait partie d'un ensemble de pratiques managériales novatrices (Figure7) regroupant la gestion concertée et la gestion collective des approvisionnements. Ces pratiques entrent dans le cadre des stratégies d'intégration de la chaîne d'approvisionnement qui permettent selon Lambert (1998) de saisir les synergies d'une gestion intra et inter entreprises ce qui renforce, à notre sens, les liens avec les concepts de partenariat, d'alliance stratégique et de réseautage organisationnel (Harland, 1996; Bechtel et Jayaram, 1997).

Selon Martin(1998), elle est une stratégie de partenariat modifiant le processus conventionnel de réapprovisionnement basé sur les bons de commandes, car les besoins d'approvisionnement sont déterminés sur la base de la demande des consommateurs, idéalement à partir des sorties caisses, et faute de mieux à partir des sorties entrepôts. Dans cette démarche, les fournisseurs et les clients gèrent ensemble l'approvisionnement des magasins des premiers et des entrepôts des seconds, en se basant sur la transmission quotidienne de données via le SICL. Ces données portent sur l'état des stocks (lieu, quantité, écarts, code produit, ruptures, mouvements ...) les dates, et les ventes. A partir de ces données et des opérations promotionnelles programmées, les fournisseurs peuvent affiner leurs prévisions pour optimiser la production et la logistique et améliorer le taux de service.

La GPA, est considérée aussi comme une pratique logistique ECR permettant de passer d'un flux poussé dans la chaîne d'approvisionnement à un flux tiré par la demande consommateur, (Debuchy, 2000). Néanmoins, sa mise en œuvre nécessite de replacer la logistique comme une fonction stratégique, car son champ d'application couvre l'ensemble de la SC, des chaînes de production chez les industriels jusqu'à la mise en rayon chez les distributeurs.

\begin{tabular}{||l|c|c|c|}
\hline \multicolumn{3}{|c}{ Focalisation croissante sur l'efficacité des réapprovisionnements } \\
\hline Principales innovations & Systèmes verticaux contractualisés & JAT et quick réponsese & ECR \\
\hline Le temps & $1950-1969$ & $\begin{array}{l}\text { Accentuation du processus } \\
\text { d'intégration entre les } \\
\text { membres du réseau }\end{array}$ & $\begin{array}{l}\text { Identification de la demańde } \\
\text { sur le marché et amélioration } \\
\text { de l'efficacité des opérations } \\
\text { promotionnelles }\end{array}$ \\
\hline $\begin{array}{l}\text { Début de recherche de } \\
\text { l'efficacité des }\end{array}$ & Déapprovisionnements & Développement de la GPA & \\
\hline
\end{tabular}

Source: Brockman 1999

Figure 7 : Evolution de la GPA

Dans la pratique, et par rapport au positionnement des opérateurs dans la SC nous distinguons, la GPA amont, la GPA aval et la GPA mutualisée (Tableau 3) qui cherche à rendre ses avantages accessibles à un grand nombre d'industriels (Abaisser le niveau de stock sur l'ensemble de la filière, contenir les coûts logistiques en optimisant le transport et gérer des unités de livraison en camions complets)

\begin{tabular}{|l|l|c|c|c|c|}
\cline { 3 - 5 } \multicolumn{2}{|c|}{} & $\begin{array}{c}\text { Nombre } \\
\text { Palette au Sol }\end{array}$ & $\begin{array}{c}\text { Nombre } \\
\text { livraisons }\end{array}$ & $\begin{array}{c}\text { Nombre Palettes } \\
\text { au Sol /Livraison }\end{array}$ & $\begin{array}{c}\text { Taux de } \\
\text { remplissage }\end{array}$ \\
\hline \multirow{2}{*}{$\begin{array}{l}\text { Fonctionnement si industriels } \\
\text { seuls }\end{array}$} & Industriel 1 & 1347 & 354 & 4 & $12 \%$ \\
\cline { 2 - 6 } & Industriel 2 & 1784 & 268 & 7 & $20 \%$ \\
\cline { 2 - 6 } & Industriel 3 & 177 & 89 & 2 & $6 \%$ \\
\cline { 2 - 6 } & Industriel 4 & 3311 & 377 & 9 & $27 \%$ \\
\cline { 2 - 6 } & Industriel 5 & 15002 & 949 & 16 & $48 \%$ \\
\cline { 2 - 6 } & Total & $\mathbf{2 1 6 2 1}$ & $\mathbf{2 0 3 7}$ & $\mathbf{1 1}$ & $\mathbf{3 2 \%}$ \\
\hline $\begin{array}{l}\text { Fonctionnement type GPA avec } \\
\text { synchronisation }\end{array}$ & Au global & $\mathbf{2 1 6 2 1}$ & $\mathbf{7 4 3}$ & $\mathbf{2 9}$ & \\
\hline
\end{tabular}


En fait, le recours à cette démarche pour la gestion des interfaces des trois entreprises mentionnées dans le tableau ci-dessus a autorisé une économie d'environ $1,2 \%{ }^{(3)}$ de leurs CA. Les objectifs de la stratégie GPA sont multiples, nous retenons la recherche d'une meilleure réponse aux attentes des consommateurs, l'accroissement de l'efficacité des flux logistiques, la désynchronisation des flux commerciaux et logistiques afin de permettre au flux d'approvisionnement de s'appuyer sur les ventes réelles aux consommateurs plutôt que sur les processus de commande du distributeur ou les fréquences de passage des agents de l'entreprise dans les linéaires. Par ailleurs, la GPA cherche à mieux planifier les opérations promotionnelles en réduisant les risques de perturbations inopinées des programmes de production, ainsi que la réduction significative des stocks et l'adaptation du plan de production de l'industriel aux sorties caisses ou entrepôts du distributeur. Elle permet de réduire les stocks intermédiaires entre les points de vente et les fabricants en ne retenant que les deux types de stocks reconnus comme source de valeur ajoutée dans la "SC", notamment le stock actif (en linéaire), et le stock tampon (régulateur de la production). En pratique, le distributeur transmet quotidiennement à l'industriel et pour chacun de ses entrepôts, l'état du stock et les sorties caisses et/ou entrepôts du jour. Le réapprovisionnement est ainsi calculé au plus juste et le distributeur peut réaliser une réduction de ses stocks allant jusqu'à $50 \%$. Grâce à cette démarche, la GPA autorise également la réduction des litiges au sein de la "SC".

Dans ce type d'arrangement, le fournisseur établi les prévisions de vente et gère les approvisionnements de ses clients distributeurs. En fait, en fonction d'un horaire de transmission préétabli, le détaillant transmet au fournisseur un ensemble de données sur l'activité de ses produits, telles que les sorties entrepôts, les sorties caisses, la quantité en stock. A partir de ces données quotidiennes et des opérations promotionnelles conclues avec ses clients, le fournisseur élabore les prévisions de vente qui permettront de générer les recommandations d'approvisionnement (Figure 8). Ces recommandations sont transmises au détaillant, qui en terme de validation confirme par accusé de réception le numéro de commande et les quantités par unité de stock qui devront être livrées. Les résultats de cette approche, utilisée dans la plupart des projets pilotes de l'industrie nord-américaine, sont significatifs. Dans le cas de Heinz Canada et Oshawa Foods, la rotation des stocks a été quadruplée tout en maintenant un niveau de service de $99 \%$. De son côté, Digital Equipement Corp a réduit de $37 \%$ son niveau de stock et de $25 \%$ le coût de commande, tout en divisant par deux le recours aux heures supplémentaires et en maintenant un niveau de service de $98 \%$.

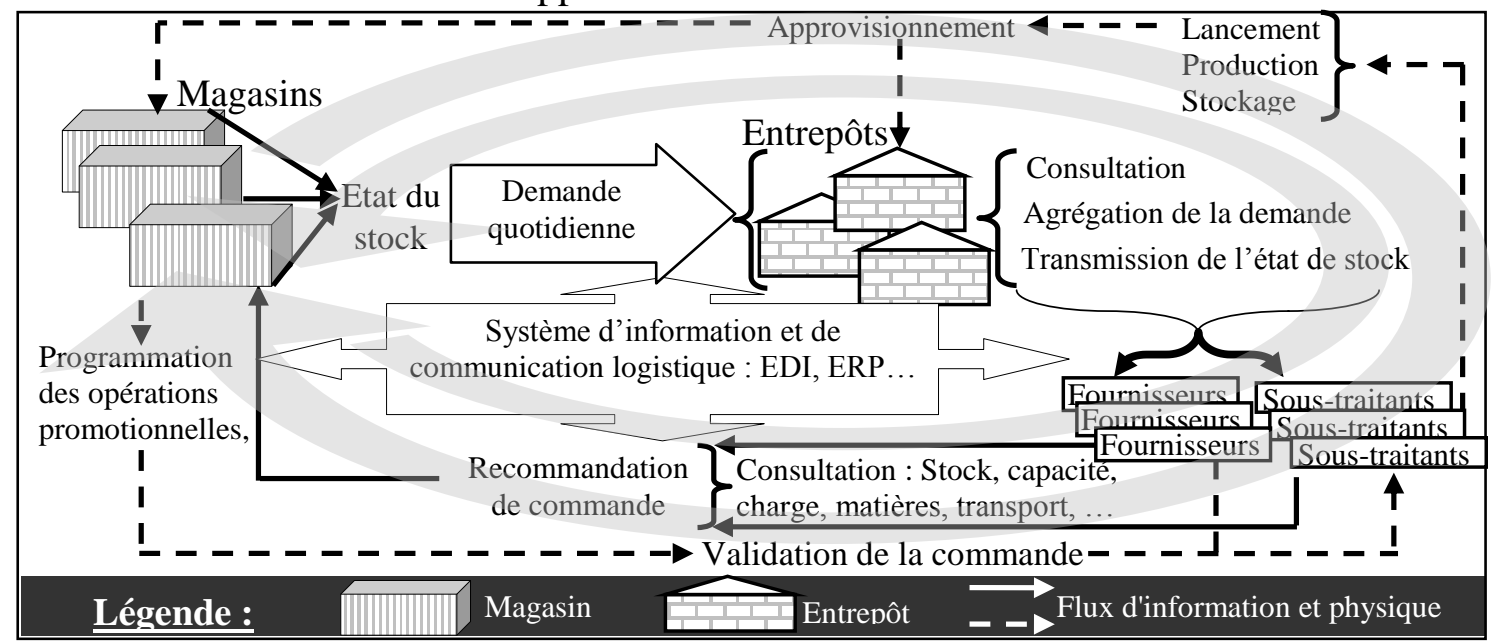

Figure 8: La GPA dans la grande distribution

${ }^{3}$ J-M. Bru, C. Pelisson \& V.Fontaine. Conférence officielle ECR 2002, Optimisation logistique multifournisseurs - multiclients et intégration des prestataires 


\section{La gestion partagée et la nature des rapports entres les partenaires industriels} 1. Le rapport entre leaders et collaborateurs en réseau, quel partage et quels gains? Ce que partagent une entreprise/pivot et ses collaborateurs, c'est essentiellement de l'information, le leader informe ses fournisseurs et sous-traitants de ses programmes d'activité et plus spécialement de ses programmes d'approvisionnement en fonction des produits qu'il a vendu et qu'il se propose de mettre en fabrication. Ainsi, en analysant l'interface industrieldistributeur de produits de grande consommation Paché (1999) constate que la cadence des approvisionnements se fait "selon un calendrier rigoureux, lui-même synchronisé au débit réel des ventes". Ce partage de l'information se concrétise par un partage du temps. En fait, en disposant des informations adéquates au bon moment (en temps réel), les fournisseurs et les sous-traitants qui travaillent en réseau, notamment dans les industries d'assemblage, peuvent agir correctement c-à-d qu'ils pourront planifier leurs flux d'approvisionnement, de production et de livraison conformément aux spécifications industrielles et logistiques du pivot. Ce dernier gère ses propres flux et fiabilise la mise à disposition de ses approvisionnements en informant ses fournisseurs et sous-traitants de ses commandes fermes et potentielles selon les délais et les modalités stipulées dans le contrat.

Quant aux gains (Tableau 4), et suite à un engagement ferme de la part des fournisseurs/soustraitants de respecter les critères imposés par le pivot et après être sélectionnés par celui-ci; le pivot s'engage à acquérir une quantité significative de composants, sur une période assez longue, ce qui garantit à ces entreprises un volume de chiffre d'affaires.

\begin{tabular}{|c|c|c|}
\hline GPA & Avantages & Inconvénients \\
\hline \multirow{5}{*}{ 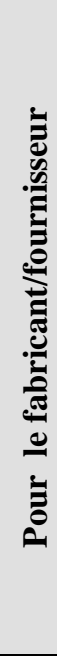 } & $\begin{array}{l}\text { Relation plus étroite avec les équipes } \\
\text { plurifonctionnelles du client. }\end{array}$ & $\begin{array}{l}\text { Investissement important au démarrage pour l'installation des } \\
\text { systèmes. }\end{array}$ \\
\hline & $\begin{array}{l}\text { Planification et gestion conjointes } \\
\text { des opérations courantes et } \\
\text { promotionnelles avec le client. }\end{array}$ & $\begin{array}{l}\text { Nécessité d'un planificateur pour gérer les programmes } \\
\text { d'approvisionnement quotidiens ou hebdomadaires. }\end{array}$ \\
\hline & $\begin{array}{l}\text { Accès aux renseignements du client } \\
\text { sur les prix promotionnels, les prix } \\
\text { vedettes, les articles stockés et les } \\
\text { ventes. }\end{array}$ & $\begin{array}{l}\text { Absence de visibilité sur les données magasin (stocks } \\
\text { disponibles, stocks en transit, stocks de sécurité, délais de } \\
\text { livraison). Les prévisions et les programmes de production n'en } \\
\text { tiennent pas compte. }\end{array}$ \\
\hline & $\begin{array}{l}\text { Fidélisation des clients du fait du } \\
\text { service apporté. Le remplacement du } \\
\text { fournisseur par le client est plus } \\
\text { difficile. }\end{array}$ & $\begin{array}{l}\text { Ajoute des étapes et du temps au processus d'approvisionnement: } \\
\text { - Transmission des données par EDI au fournisseur. } \\
\text { - Communication au client des programmes élaborés, } \\
\text { - Validation des programmes par ce dernier. }\end{array}$ \\
\hline & $\begin{array}{l}\text { Avantages supplémentaires du fait de } \\
\text { l'intégration des fournisseurs de } \\
\text { matières premières et d'emballage. }\end{array}$ & $\begin{array}{l}\text { Absence de prise en compte des contraintes capacitaires du } \\
\text { centre de distribution (en transport, réception et entreposage) }\end{array}$ \\
\hline \multirow{4}{*}{ 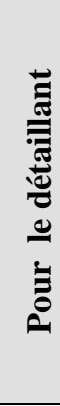 } & $\begin{array}{l}\text { Aucun besoin d'expertise interne en } \\
\text { matière de prévision et de } \\
\text { réapprovisionnement. }\end{array}$ & $\begin{array}{l}\text { L'expertise dans les systèmes de prévision et de } \\
\text { réapprovisionnement est externe, et dépend du fournisseur. }\end{array}$ \\
\hline & Coûts de démarrage négociables. & $\begin{array}{l}\text { Le réapprovisionnement des commandes des magasins n'est pas } \\
\text { inclus dans le champ d'application. }\end{array}$ \\
\hline & $\begin{array}{l}\text { Avantages immédiats en termes de } \\
\text { service client et de rotation des } \\
\text { stocks. }\end{array}$ & $\begin{array}{l}\text { Surcharges potentielles au niveau du centre de distribution et } \\
\text { des magasins. Absence de coordination centrale des expéditions } \\
\text { du fournisseur. }\end{array}$ \\
\hline & & $\begin{array}{l}\text { Possibilité d'abus de confiance pour les renseignements sur les } \\
\text { prix et les promotions. }\end{array}$ \\
\hline
\end{tabular}

Tableau 4 : les avantages et les inconvénients de la GPA

En plus, ils peuvent être gagnants si, après la signature du contrat et grâce à la mise en place d'un système d'amélioration continue, ils parviennent à qualité égale, à diminuer leurs coûts. Ces collaborateurs, peuvent également gagner en notoriété. En fait, la sélection d'une PME pour livrer un leader du secteur peut être interprétée comme une reconnaissance pouvant 
engendrer énormément d'effets bénéfiques. En plus, les collaborateurs peuvent bénéficier des aides en conception, en méthodes, en procédures, en outils... comme ils peuvent tirer avantage des solutions organisationnelles, des innovations technologiques qu'ils maîtrisent lors de leur contrat pour satisfaire aux spécifications du pivot. Ils peuvent ailleurs, acquérir une plus grande visibilité sur la demande d'où la possibilité d'adapter leurs plans de production à la demande et en retour de réduire leurs stocks de produits finis et de matières premières.

De son côté, le leader peut bénéficier d'une diminution du coût global, de l'amélioration de la productivité avec la suppression des tâches à faible valeur ajoutée et de l'optimisation des processus par la réduction des cycles et la maîtrise de la qualité. Il peut aussi bénéficier d'un stockage de pièces lui appartenant dans les locaux du fournisseur.

Les intérêts partagés ${ }^{(4)}$ portent sur une augmentation de la qualité et de la fraîcheur en linéaire, la mise en place plus rapide de nouveaux produits, une plus grande fiabilité du réapprovisionnement (Tableau 5), l'optimisation des moyens logistiques et la responsabilisation de l'approvisionneur (collaboration plus étroite, suivi de tableaux de bord, plans d'action) et donc le développement d'un climat de partenariat.

\begin{tabular}{|c|c|c|c|}
\hline \% Rupture & Global & Gels douche & Déodorants \\
\hline Avant & $\mathbf{6 , 4 \%}$ & $\mathbf{1 , 1 \%}$ & $\mathbf{8 , 5 \%}$ \\
\hline Après & $\mathbf{2 , 6 \%}$ & $\mathbf{0 \%}$ & $\mathbf{3 , 7 \%}$ \\
\hline
\end{tabular}

Source: Laurent 2002 Tableau 5: Taux de rupture avant et après la mise en œuvre de la GPA par Cora et LASCAD

\section{Mode de coordination}

La relation de partage entre la firme/pivot et ses collaborateurs est-elle basée sur la coopération et l'entente ou sur la contrainte? Deux approches peuvent être distinguées en réponse à cette question. La première, que nous appelons "réseau de confiance" considère que ce type de relation n'est obligatoirement pas formalisé par contrat, mais il résulte de l'histoire commune, de l'expérience mutuelle des organisations et il est toujours basé sur un engagement des partenaires. Elle préconise (Ghoshal et Moran 1996; Chiles et MacMackin 1996) que le mode formel de contrôle (contrat) générait un certain nombre d'effets pervers préjudiciables à la performance des structures de coopération, notamment l'apparition d'un climat de défiance entre partenaires ne faisant que renforcer les tentations opportunistes au lieu de les réduire. Même dans le cas de l'existence d'un contrat (Paché et des Garets 1997), ses clauses ne font que spécifier un niveau de performance attendu plutôt qu'elles ne décrivent la nature et les modes d'exécution des opérations.

La deuxième approche que nous appelons "réseau de contrat" considère que le partage de la gestion des approvisionnements doit obligatoirement passer par un contrat cadre. Il est censé apporter une certaine sécurité aux partenaires impliqués en limitant considérablement les risques d'opportunisme et de trahison, encouragés par l'incomplétude des contrats (Williamson, 1985), elle-même conséquence logique de l'information imparfaite et de la rationalité limitée des acteurs. La théorie des coûts de transaction rejette ainsi, l'éventualité de la confiance dès lors qu'un risque menace la relation d'échange et propose le recours à l'intégration verticale pour se prémunir de l'opportunisme des acteurs. La création de réseaux de fournisseurs, pouvant s'assimiler à une quasi-intégration des fournisseurs sur le site de production serait, par l'émergence "d'otages", un moyen de déjouer l'opportunisme des fournisseurs en enfermant les relations dans un semblant d'hiérarchie.

Toutefois, une position intermédiaire (Baudry, 1995), combine confiance et contrat. L'auteur, considère que la première est l'une des composantes essentielles au fonctionnement d'un réseau, mais son origine ne peut être que l'existence du second, car "contracter avec

${ }^{4}$ G. Debuchy \& B. Mary; Gestion partagée des approvisionnements Aval; Conférence officielle ECR 2000 
quelqu'un, c'est déjà un acte de confiance". La confiance est nécessaire dès lors qu'un risque est lié à l'échange. La confiance en un partenaire suppose donc qu'on lui attribue une probabilité de risque (liée à l'échange) très faible. Il considère que le recours à la hiérarchie (coûts de transaction) génère des coûts de contrôle élevés et ne garantit nullement l'absence d'opportunisme des acteurs, ni même de celui des contrôleurs.

\section{Pratiques et perspectives.}

1. La GPA dans le secteur de l'automobile : vers les fournisseurs immédiats.

Le mouvement de partage des approvisionnements a vu le jour dans le secteur de l'automobile avec le mouvement de "désintégration verticale" des années 80 (Fassio, 2000). Actuellement, en Europe et au Japon, $70 \%$ de la valeur ajoutée à une automobile le serait par des entreprises autres que le concepteur-assembleur. En conséquence, ces entreprises ont adoptés une stratégie de recentrage sur leurs métiers de base, conception et assemblage final.

Dans ce secteur, un fournisseur/sous traitant ne peut qu'accepter la charge de travail que le pivot lui propose. En refusant, il se prive forcément d'une fraction importante du chiffre d'affaires. Le cas de Former, équipementier en fixations pour l'automobile, est fortement édifiant. Après avoir refusé de coopérer avec Renault en 1997, Former se repent en 1999 après avoir accumulé une perte annuelle de chiffre d'affaires de 35 millions de FF (5335 715 $€$ environ). Mieux encore, même si un véhicule sur trois fabriqués en Europe est habillé par les aciers de Sollac (chiffre d'affaires consolidé 1997 : 37,8 milliards de FF soit environ 5,76 milliards $€$ ), son directeur logistique estime que l'entreprise ne peut se permettre de refuser les règles imposées par les donneurs d'ordre de la taille de Renault ou de Peugeot.

L'industrie automobile s'oriente actuellement vers une réorganisation totale du processus de conception/industrialisation où les constructeurs tendent à réduire drastiquement le nombre de fournisseurs/sous traitants (équipementiers de premier rang). Pour un groupe comme P.S.A., le nombre de fournisseurs est passé en une quinzaine d'années d'environ 1000 à 380 fournisseurs, dont 18 assurent 50\% des achats. De même dans l'usine MCC d'Hambach, sept équipementiers sont intégrés au site de montage et fournissent la grande partie des composants du véhicule Smart. ${ }^{(5)}$

La mise en réseau de la conception et de l'industrialisation pousse les constructeurs vers la recherche d'une plus grande maîtrise des coûts accompagnée par une réduction du temps de développement des nouveaux modèles et du temps de réponse aux commandes des clients. Si le développement de la Renault Mégane a demandé 60 mois, celui de la Clio II n'a requis que 40 mois et le constructeur espère réduire ce temps à 30 mois pour les prochains modèles.

Pour atteindre cet objectif, de nouveaux efforts en gestion de production et en organisation devront être effectués, notamment en réduisant les délais d'approvisionnement, composante principale du temps d'attente (et d'arrêt) sur les lignes de montage. En fait, la sécurisation des approvisionnements peut être assurée au niveau logistique par la création des parcs industriels fournisseurs permettant de gérer la diversité au plus près de la chaîne de montage.

Dans le cas de la Smart ${ }^{(6)}$ nous avons assisté à une évolution de la gestion partagée des approvisionnements vers une gestion intégrant les fournisseurs immédiats. En fait, la plateforme de production est cogérée par l'ensemble des intervenants. Les équipementiers

\footnotetext{
${ }^{5}$ S. Renault \& B. Morisse, "L’entreprise étendue ou les bénéfices d'un partenariat de proximité". Troisième RIRL. Québec. Canada.

${ }^{6}$ Le projet SMART (SWATCHMOBILE) est né en 1989 d'un accord commun entre SWATCH et VOLKSWAGEN. Après le retrait de ce dernier en 1992, le projet a été relancé par MERCEDES-BENZ et SMH et a conduit à la création, en avril 1994 de MICRO CAR COMPACT (MCC) détenue à 51\% par MERCEDESBENZ et 49\% par SMH. Depuis décembre 1997, SMH a baissé sa participation dans MCC à seulement 19\% pour finalement quitter cette société moins de onze mois plus tard.
} 
financent eux-mêmes la réalisation des outillages ainsi qu'une grande partie du projet ${ }^{(7)}$ par rapport à la contribution de la firme MCC qui ne dépasse pas $8 \%$ de la valeur ajoutée d'un véhicule. Cette stratégie, met les équipementiers dans une situation "d'otage" vis-à-vis du pivot et devrait donc amincir sensiblement toute tentation opportuniste. Les investissements irrécupérables sont importants pour chacun des partenaires ce qui constitue un gage de respect des engagements mutuels. La perte que générerait toute tentative de trahison est supérieure aux gains qui pourraient être récoltés à cette occasion.

Concrètement, les fournisseurs sont présents sur les lignes de montage et les sept fournisseurs du premier rang garantissent la fourniture de composants principaux que sont la carrosserie, le cockpit tout équipé, l'essieu arrière (avec transmission), le module avant (avec éclairage et radiateur), les portes et éléments d'habillage intérieur. Ils fournissent ainsi $90 \%$ du matériel qui arrive sur la ligne de montage. Ces équipementiers rassemblent eux-mêmes une trentaine de fournisseurs de second niveau et effectuent directement l'assemblage des pièces sur les chaînes du constructeur.

En conséquence, la reconfiguration de la chaîne logistique enquête d'une meilleure réactivité a donné naissance, dans l'industrie automobile à un nouveau rang de fournisseurs : les fournisseurs immédiats.

\section{Cas des produits de grande consommation et de la grande distribution}

En matière de partenariat Industrie-Commerce articulé autour de la GPA, les expériences de Carrefour avec Amora Maille et Coca cola Entreprise sont avant-gardistes.

Amora Maille assure les trois quarts de la production française de la Moutarde et couvre $50 \%$ du marché des condiments. Les matières premières et les emballages représentent $75 \%$ du coût de revient des produits élaborés. Par conséquent, la réduction des stocks en amant (matières premières) et en aval (produits finis), via la maîtrise de la chaîne d'approvisionnement est devenue un objectif du premier ordre.

L'action sur les stocks des produits finis s'est concrétisée par la mise en œuvre d'une démarche GPA avec le distributeur Carrefour. Concrètement, Amora Maille a procédé à une révision de l'ensemble des processus de planification impactant les fonctions prévisions de vente, logistique, production et achat. Au sein de la direction logistique, une direction des flux est crée en 1998 et une équipe est affectée à la gestion des approvisionnements des clients dont six personnes ont été chargées strictement et expressément des activités de la GPA et du suivi de la relation logistique avec les clients. Quatre mois après le lancement de l'opération $15 \%$ des ventes étaient gérés en GPA, après une année et demi ce taux a atteint $40 \%$.

Les objectifs majeurs (Debuchy, 2000), escomptés de la mise en place de cette démarche à l'interface des deux partenaires sont l'amélioration du taux de service via une meilleure connaissance de la demande et une réactivité accrue (délai de livraison), la baisser du niveau des stocks via des techniques comme le lissage des flux et la fiabilisation des échanges par le développement des messages EDI. Les résultats de cette mise en œuvre sont concluants :

$\checkmark$ Réduction des stocks de produits finis de matières premières et emballages. Les stocks dans les entrepôts des clients (Carrefour) ont été divisés par deux.

$\checkmark$ Le taux de service a pu atteindre $100 \%$ pour toute l'activité de l'entreprise pendant plusieurs jours de suite. Ce taux a atteint pour le client Carrefour 99,9\%

$\checkmark$ Le taux de litiges avec le client Carrefour a été divisé par dix

$\checkmark$ Le chiffre d'affaires s'est amélioré grâce à une meilleure disponibilité des produits dans les linéaires et l'image de l'entreprise s'est améliorée.

$\checkmark$ Sur le plan organisationnel, les relations entre acheteurs et vendeurs se renforcent par l'émergence d'une collaboration entre logisticiens et informaticiens des deux entreprises.

\footnotetext{
${ }^{7}$ Les équipementiers ont apporté $60 \%$ des 2,5 milliards de FF, environ 3,8 millions $€$, d'investissements annoncés sur le site
} 
De son côté, Carrefour tente de généraliser cette démarche, en pratiquant la GPA pour la moitié de ses produits d'épicerie avec une trentaine d'industriels. En plus, $60 \%$ du CA sur les produits de Grande Consommation est géré en GPA.

Dans ce sens, carrefour à lancé fin $1998^{(8)}$ un autre pilote GPA avec Coca Cola Entreprise (CCE) chargé de l'embouteillage et la distribution pour l'Europe. Ce pilote, consistait pour CCE à prendre directement en charge le stock de ses produits chez carrefour et à gérer à distance l'approvisionnement et la mise en linéaire des nouveaux produits dans les meilleurs délais. Actuellement (2004) environ 50\% du chiffre d'affaires grande distribution de CCE passe par la GPA. L'objectif étant d'atteindre le taux de $70 \%$ en 2005 et d'étendre la démarche vers d'autres types de réseaux de distribution, notamment vers les activités "hors foyer" en l'occurrence les hôtels, les restaurants, et les bars.

En fin, la mis en œuvre des recommandations de l'ECR France a permet une économie moyenne pour la chaîne au niveau de l'interface industriels et distributeur équivalente à $23 \%$ du coût de l'interface dont 1,8\% grâce à la GPA (Tableau 6). Ce gain peut atteindre 3,4\% pour des partenaires appliquant $100 \%$ de la solution à $100 \%$ d'un flux.

\begin{tabular}{|l|c|c|}
\hline Composants de l'interface & $\begin{array}{l}\text { Gains pour l'ensemble } \\
\text { de la chaîne }\end{array}$ & $\begin{array}{l}\text { Gains pour des partenaires appliquant } \\
100 \% \text { de la solution à 100\% d'un flux }\end{array}$ \\
\hline Entrepôt partagé / regroupement des flux & $0,5 \%$ & 1,1 \\
\hline Cross Doking / Fréquence de livraison stable & $0,4 \%$ & 1,2 \\
\hline GPA Centre de Distribution & $\mathbf{1 , 2 \%}$ & $\mathbf{2 , 3}$ \\
\cline { 1 - 3 } GPA Points de Vente & $\mathbf{0 , 6 \%}$ & $\mathbf{1 , 1}$ \\
\hline Bonne gestion des hausses des tarifs & $0,5 \%$ & 0,5 \\
\hline Planning communs & $0,2 \%$ & 0,25 \\
\hline Prévisions concertées & $0,35 \%$ & 0,4 \\
\hline Suppression des contrôles à la réception & $0,3 \%$ & 0,4 \\
\hline Total & $4,05 \%$ & $7,25 \%$ \\
\hline
\end{tabular}

Source: Debuchy 2000 Tableau 6 : gains réalisés au niveau de l'interface industriels distributeurs

\section{La gestion des approvisionnements dans les entreprises au Maroc}

Dans un objectif d'exploration des pratiques et de la place de la gestion collaborative des approvisionnements sous toute ses formes dans les entreprises marocaines, nous avons lancé une étude auprès de six entreprises opérant dans les secteurs de l'agroalimentaire, les composants automobile, le petit électroménager, l'électronique et la chimie. Nous avons choisi ces entreprises en tenant compte de la diversité des secteurs d'activités, de leur représentativité en terme de taille (petites, moyennes et grandes) et des facteurs capital et chiffre d'affaires significatifs par rapport à leurs secteurs respectifs.

L'étude a débuté en Août 2003 et a durée jusqu'à la fin de Mars 2004. Pendant cette durée nous avons surmonté de nombreux obstacles, notamment, l'accès à certaines entreprises, le "bon interlocuteur" n'était pas toujours disponible et l'absence dans plusieurs cas de données chiffrées et précises. Cette difficulté d'accéder à une information précise, fiable et pertinente, nous impose en conséquence, beaucoup de précautions et une très grande relativité quant à la généralisation des résultats de cette étude.

Au niveau des résultats, nous retenons que dans $66,7 \%$ des cas la gestion des achats et des approvisionnements est assurée par une fonction nommée généralement achat/approvisionnement, elle-même attachée à la direction financière, à la direction logistique ou à la direction générale. L'effectif de cette fonction varie entre 5 et 8 personnes dans 33,3\% des cas et ses principaux objectifs consistent dans l'achat au meilleur prix, l'assurance de la qualité des produits et des services achetés, la recherche de nouveaux fournisseurs et la maîtrise du niveau des stocks.

\footnotetext{
${ }^{8}$ F. Deblock de JDNet Solution (Benchmark Group) \& I. Bellaïche de Coca Cola Entreprise le 20/01/ 2004
} 
Quant à la position de la fonction au sein de la structure, $50 \%$ des entreprises considèrent que la fonction a un caractère "stratégique". Toutefois, nous constatons que seulement dans $16,7 \%$ des cas (Tableau 7) une concordance existe entre l'aspect "stratégique" présumé de la fonction et la participation de son responsable à l'élaboration et à la mise en ouvre des stratégies de l'entreprise.

\begin{tabular}{|l|l|c|l|l|l|}
\hline Participation decision & Non-réponse & $\begin{array}{c}\text { Elaboration et } \\
\text { mise en oeuvre }\end{array}$ & $\begin{array}{c}\text { Seulement à la } \\
\text { mise en ouvre }\end{array}$ & Consulté & Total \\
\hline Non réponse & $16,7 \%$ & $16,7 \%$ & $00 \%$ & $16,7 \%$ & $50 \%$ \\
\hline Stratégique & $16,7 \%$ & $\mathbf{1 6 , 7 \%}$ & $16,7 \%$ & $00 \%$ & $50 \%$ \\
\hline Total & $33,3 \%$ & $33,3 \%$ & $16,7 \%$ & $16,7 \%$ & $100 \%$ \\
\hline
\end{tabular}

Tableau 7: Positionnement stratégique et participation du responsable approvisionnement à la prise de décisions

Par ailleurs, 33,3\% des entreprises disposent d'une stratégie de gestion des fournisseurs mais $16,7 \%$ seulement cherchent à réduire le nombre des fournisseurs et instaurer des relations de partenariat. (Tableau8).

En outre, $50 \%$ des entreprises informent leurs fournisseurs de leurs prévisions et 33,3\% programment des visites aux locaux de ces derniers. Toutefois, aucune exigence de certification n'est imposée, aucun échange de personnel n'est envisagé, aucun investissement financier chez eux n'a été programmé, aucune entreprise n'est impliquée dans une démarche ECR. Seulement 16,7\% effectuent des réunions périodiques avec leurs fournisseurs, leurs font systématiquement des propositions de livraison et leurs dispensent une certaine formation.

\begin{tabular}{|l|c|c|l|l|}
\hline \multicolumn{1}{|c|}{ Réduction des fournisseurs } & Non réponse & Oui & Non & Total \\
Stratégie fournisseurs & $16,7 \%$ & $00 \%$ & $16,7 \%$ & $33,3 \%$ \\
\hline Non-réponse & $00 \%$ & $\mathbf{1 6 , 7 \%}$ & $16,7 \%$ & $33,3 \%$ \\
\hline Oui & $00 \%$ & $00 \%$ & $33,3 \%$ & $33,3 \%$ \\
\hline Non & $16,7 \%$ & $16,7 \%$ & $66,7 \%$ & $100 \%$ \\
\hline Total & &
\end{tabular}

Tableau 8 : Stratégie de gestion des fournisseurs

Quant aux critères de sélection des fournisseurs, nous retenons la prédominance du critère "prix" qui arrive entête loin devant la qualité, le service après vente, et le délai de livraison.

Le système d'information des entreprises est faiblement équipé en moyens informatiques (Tableau 9). Dans 33,3\% des cas les stocks représentent plus d'un tiers du total bilan de l'exercice 2002. En aucun cas des méthodes de calcul économique ne sont utilisées et $50 \%$ des entreprises utilisent un réapprovisionnement en fonction des besoins (coup par coup). 16,7\% des cas prétendent avoir une cellule composée du personnel de l'entreprise et de celui du fournisseur destinée exclusivement à la gestion et la coordination des approvisionnements et disposent d'un accès libre aux informations concernant les stocks, mais aucun accès n'est autorisé aux sorties caisses. Dans la même proportion les entreprises estiment qu'ils ont un système de gestion partagée des approvisionnements avec les fournisseurs et avec les clients. Toutefois, aucune information concernant le pourcentage des achats et des ventes effectuées selon cette modalité n'a pu être récupéré.

\begin{tabular}{|l|c|c|c|c|c|c|c|c|}
\hline & & & \multicolumn{5}{|c|}{ Logiciels de gestion } \\
\hline Réponse & ERP & Code barre & EDI & CAO $^{*}$ & Entrepôt & Stock & Prévisions & Transport \\
\hline Non - réponse & $80 \%$ & $66,7 \%$ & $100 \%$ & $0 \%$ & $50 \%$ & $33,3 \%$ & $66,7 \%$ & $50 \%$ \\
\hline Oui & $0 \%$ & $33,3 \%$ & $0 \%$ & $83,3 \%$ & $16,7 \%$ & $50 \%$ & $0 \%$ & $16,7 \%$ \\
\hline Non & $20 \%$ & $0 \%$ & $0 \%$ & $16,7 \%$ & $33,3 \%$ & $16,7 \%$ & $33,3 \%$ & $33,3 \%$ \\
\hline Total & $100 \%$ & $100 \%$ & $100 \%$ & $100 \%$ & $100 \%$ & $100 \%$ & $100 \%$ & $100 \%$ \\
\hline
\end{tabular}

Tableau 9: utilisation des techniques et logiciels de gestion dans les entreprises marocaines. 


\section{Conclusion:}

L'objectif de ce travail était la mise en évidence du rôle capital des techniques d'approvisionnement collaboratives dans l'optimisation des flux au sein de la SC. Dans cette perspective, nous avons articulé la première partie autour des soubassements théoriques des stratégies relationnelles, du partenariat industriel et des formes organisationnelles réticulaires. Nous considérons ces trois facteurs comme une réaction stratégique et structurelle face à l'évolution de l'environnement vers plus de compétitivité et face aux besoins grandissant de synchronisation des flux de la SC. Cette quête de synchronisation des flux physiques, informationnels et financiers, a constitué à nos yeux, une forte plate-forme de développement de nouvelles démarches en management telle que l'ECR et un facteur central du développement du SICL.

En suite, nous avons présenté les techniques de réapprovisionnement continue avec une focalisation particulière sur la GPA. Cet intérêt est justifié non seulement par sa mise en œuvre de plus en plus fréquente ou par les gains qu'elle autorise, mais surtout par son champ d'action qui couvre l'ensemble de la SC et qui, de facto, revalorise la logistique en la replaçant comme une fonction stratégique. En fait, nous considérons que le succès des techniques du RC n'est nullement une simple opération technique, mais la résultante d'un processus consensuel mettant en avant des valeurs de partage et des modes de coordination entres les différents acteurs de la SC.

Ensuite, nous avons relevé la tendance du secteur de l'automobile vers le développement des fournisseurs immédiats et le recours à la GPA dans la grande distribution comme un levier incontournable pour l'amélioration $\mathrm{du}$ taux de service tout en réduisant les stocks. L'amélioration de ce taux doit être soutenue par le développement de la réactivité des différents acteurs de la chaîne logistique et par une plus grande fiabilisation des échanges d'où une autre fois le rôle prépondérant du SICL.

En dernier lieu, nous retenons qu'au Maroc la gestion des approvisionnements ne revêt un caractère stratégique que pour une minorité des entreprises et que la démarche ECR et le plan directeur d'approvisionnement sont quasiment inexistants dans leurs systèmes de management. Nous avons relevé, aussi que la majorité utilise un systèmes de réapprovisionnement en fonction des besoins (coup par coup). Toutefois, des pratiques entrant dans le cadre de la GPA fonds leur émergence dans certaines structures. Malgré les réserves que nous pouvons formuler face à ces résultats, nous considérons qu'ils doivent, interpeller fortement les acteurs dans les entreprises marocaines qui se trouvent -mondialisation obligeau plein milieu d'un processus de libéralisation irréversible et impitoyable. 


\section{Bibliographie :}

Alban, D., 1997, "Management du système d'information et politiques relationnelles d'organisation réticulaire", Cahier de Recherche.01, IAE de PARIS, GREGOR.

Alcouffe, C. et Correge, N., 1999, "Evaluation des performances dans les organisations en réseaux de sous-traitants :l'exemple d'aerospatiale-Matra Airbus, Revue Française de gestion Industrielle", Vol.18, $\mathrm{N}^{\circ} 4$.

Altersohn, C., 1992, "De la sous-traitance au partenariat industriel, Dynamiques d'Entreprises", l'harmattan.

Anastassopoulos, J.P., et all, 1988, "STRATEGOR, Stratégie, structure, décision, Identité : Politique générale d'entreprise". InterEdition.

Baudry, B. \& Bouvier-patron .P, 1994, "Génie industriel" - CH : de la sous-traitance traditionnelle à la sous-traitance partenariale : une application de la théorie de l'agence. PUG Baudry. B., 1995, "L'économie des relations interentreprises", Editions La Découverte, Paris. Bechtel, C.\& Jayaram, J. 1997 «Supply Chain Management : A Strategic Perspective», International Journal of Logistics Management, vol. 8, $\mathrm{n}^{\circ} 1$.

Benchimol, G., 1993, "L'entreprise étendue". Hermes.

Brockman, B., et Morgan,R., 1999, "The evolution of managerial innovations in distribution :what prospects for ECR?", International journal of retail \& Distribution Management, Vol.27, $\mathrm{n}^{\circ} 10$.

Chiles, T. H. and MacMackin .J. F., 1996, "Integrating Variable Risk Preferences, Trust and Transaction Cost Economics", Academy of Management Journal, 21(1).

Colin, J., 1996, "La logistique : histoire et perspectives", in La logistique, recherches et mise en œuvre. Coordination Gourgand, Lièvre, Edition Hermes.

Colin, J, 1997, "La logistique amont : de la maîtrise des interfaces avec les fournisseurs à la conception des produits".In Management logistique, une approche transversale. Editions Litec. Dornier, P.P., 1991, "Plein flux sur l'entreprise". Nathan.

Dumoulin, R., Meschi. P-X. et Uhlig. T., 2000, "Socialisation, contrôle et performance dans les réseaux d'alliances logistiques", Troisième Rencontre Internationale de Recherche en Logistique. Quebec. Canada.(RIRL. 2000)

Fabbe-C,N., 1998," Pour un pilotage global des flux", Ambitions Sud International, n 10, mai.

Fassio, G., 2000, "Quels partages entre acteurs de l'interface « approvisionnement » dans les secteurs de l'aéronautique et de l'automobile ?" Troisième RIRL. Quebec. Canada.

Gallo, M. et Patricot, H., 2002, Conférence officielle, ECR France.

Ghoshal, S. \& Moran, P., 1996, " Bad for Practice : a Critique of the Transaction Cost Theory", Academy of Management Journal, 21(1).

Giget, M., 1998, "La dynamique stratégique de l'entreprise", Paris, Dunod.

Gulati, R., 1998, "Alliances and Networks", Strategic Management Journal, 19 (2).

Hamel, G., \& Prahalad, C.K., 1995, "La conquête du Futur", Paris, ERPI.

Harland, C.M., 1996, "Supply Chain Management : Relationship, Chains and Networks", British Journal of Management, vol. 7, Special Issue.

Jouanneau, J. \& Galand, L., 2002 "ECR bilatéral, Catégorie ultra - frais". Conférence officielle, ECR France.

Juga, J., 1996, "Organizing for Network Synergy in Logistics", International Journal of Physical Distribution \& Logistics Management, vol. 26, $\mathrm{n}^{\circ} 2$.

Lambert, D.M., Emmelhainz M.A, \& Gardner, J.T., 1996 "Developping and Implementing Supply Chain Partenership", The International Journal of Logistics Management, vol. 7, no 2. Lambert, D.M., Stock, J.R. \& Elram, L.M., 1998, "Fundamentals of Logistics management", Irwin-McGraw-Hill.

Laurent, S. \& Philippe, R., 2002, Pilote CORA- LaSCAD, conférence officielle, ECR France. 
Lievre, P., 2000, "Système d'information logistique : circulation d'information rationnelle et/ou relationnelle ?", Troisième RIRL. Québec. Canada. .

Martin, A.J. \& Landvater, D.V., 1998, "Principes et perspectives du réapprovisionnement continu au coeur de la supply chain", ASLOG.

Miles, R.E. \& Snow, C.C., 1984, "Fit, Failure and the Hall of Fame", California Management Review, Spring, 10-28.

Miles, R.E. \& Snow, C.C., 1992, "Causes of Failure in Network Organizations", California Management Review, Summer, 53-72.

Paché, G., 2000, "Repérer les évolutions du canal logistique: quelques enjeux majeurs dans une perspective marketing", Convegno 'LE TENDENZE DEL MARKETING IN EUROPA' Università Ca' Foscari Venezia 24 Novembre.

Paché, G., \& Paraponaris, C., 1993, "L’entreprise en réseau". Que sais-je ?.

Paché, G., \& DesGarets. V., 1997, "Relations inter-organisationnelles dans les canaux de distribution: les dimensions logistiques", Recherche et Applications en Marketing, vol.12, n². Paché, G., 1999, "La logistique comme technique des flux. Une source de pouvoir aux mains des distributeurs", Science et technologie, regards croisés, CNRIUT, Ed. l'Harmattan.

Perraudin, X. \& Mignot. D., 2000, Conférence officielle, ECR France.

Porter, M., 1986, "L'avantage concurrentiel", InterEditions.

Richardson, G.B., 1972, "The Organization of Industry", Economic Journal, September.

ROY, J. \& BIGRAS, Y., 2000, "Le partenariat: un élément clé de la chaîne logistique". Troisième RIRL. Québec. Canada.

Simon, H., 1961, "Administrative behaviour", 2ème édition, New-York, Mac Millan.

Thorelli, H., 1986, "Networks :between markets and hierarchies", Strategic Management Journal, vol.7, $\mathrm{N}^{\circ} 1$.

Williamson, O.E., 1985, "The Economic Institutions of Capitalism, Firms, Markets, Relational Contracting", New-York, Free Press. 